\title{
Two new mathematical models for prediction of early mortality risk in coronary artery bypass graft surgery
}

\author{
Alireza Alizadeh Ghavidel, MD, ${ }^{a}$ Hoda Javadikasgari, MD, ${ }^{a}$ Majid Maleki, MD, ${ }^{\mathrm{b}}$ Arsha Karbassi, MD, \\ Gholamreza Omrani, MD, ${ }^{\mathrm{a}}$ and Feridoun Noohi, $\mathrm{MD}^{\mathrm{b}}$
}

Objectives: The aim of this study was to develop new models for prediction of short-term mortality risk in on-pump coronary artery bypass grafting $(\mathrm{CABG})$ surgery using decision tree (DT) methods.

\begin{abstract}
Methods: Between September 2005 and April 2006, 948 consecutive patients underwent CABG surgery at Rajaie Heart Center. Potential risk factors were reviewed and univariate and multivariate analysis for short-term mortality were performed. The whole dataset was divided into mutually exclusive subsets. An entropy error fuzzy decision tree (EEFDT) and an entropy error crisp decision tree (EECDT) were implemented using $650(68.6 \%)$ patient data and tested with $298(31.4 \%)$ patient data. Ten times hold-out cross validation was done and the area under the receiver operative characteristic curve (AUC) was reported as model performance. The results were compared with the logistic regression (LR) model and EuroSCORE.
\end{abstract}

Results: The overall short-term mortality rate was $3.8 \%$, and was statistically higher in women than men $(P<.001)$. The final EEFDT selected 19 variables and resulted in a tree with 39 nodes, 20 conditional rules, and AUC of $0.90 \pm 0.008$. The final EECDT selected 15 variables and resulted in a tree with 35 nodes, 18 conditional rules, and AUC of $0.86 \pm 0.008$. The LR model selected 10 variables and resulted in an AUC of $0.78 \pm 0.008$; the AUC for EuroSCORE was $0.77 \pm 0.003$. There were no differences in the discriminatory power of EEFDT and EECDT $(P=.066)$ and their performance was superior to LR and EuroSCORE.

Conclusions: EEFDT, EECDT, LR, and EuroSCORE had clinical acceptance but the performance and accuracy of the DTs were superior to the other models. (J Thorac Cardiovasc Surg 2014;148:1291-8)

Supplemental material is available online.

Mortality risk evaluation has been increasingly emphasized in cardiac surgery. ${ }^{1}$ The aims of developing risk models include quality monitoring of surgical performance, counseling patients and deciding treatment, cost-benefit analysis, or all of these purposes. Coronary artery bypass grafting $(\mathrm{CABG})$ is one of the most common cardiac surgeries and most of the candidate patients are old with comorbidities. Thus, accurate prediction of operative risk is critical for patients and doctors to make a proper informed decision about surgery. ${ }^{2}$

\footnotetext{
From the Heart Valve Disease Research Center, ${ }^{a}$ Rajaei Cardiovascular Medical and Research Center; Department of Cardiology, ${ }^{\mathrm{b}}$ Shahid Rajaie Heart Hospital; Interventional Cardiology, ${ }^{\mathrm{c}}$ Tehran Heart Center, Tehran, Iran.

Disclosures: Authors have nothing to disclose with regard to commercial support.

Received for publication Aug 4, 2012; revisions received Sept 1, 2013; accepted for publication Feb 3, 2014; available ahead of print March 7, 2014.

Address for reprints: Hoda Javadikasgari, MD, Heart Valve Disease Research Center, Rajaei Cardiovascular Medical and Research Center, Vali-Asr Ave, Niyayesh Blvd, Tehran 199691-1151, Iran (E-mail: hoda.javadi.k@gmail.com). $0022-5223 / \$ 36.00$

Copyright (c) 2014 by The American Association for Thoracic Surgery http://dx.doi.org/10.1016/j.jtcvs.2014.02.028
}

Several cardiac surgery risk models have been proposed. ${ }^{3,4}$ The EuroSCORE model has been shown to have the highest discriminatory power among all models; its area under the receiver operating characteristic (ROC) curve (AUC) did not exceed 0.78 .5

Most risk scoring systems, like EuroSCORE, have been developed based on assumption of a linear relationship among variables. Therefore, there is a need to evaluate newer methods with more complex mathematical assumptions. Decision trees (DTs) are popular reasoning methods and have been successfully applied in clinical decision making. For example, a DT has recently been used to determine the most appropriate method of rectal cancer management. ${ }^{6}$ Moreover, it has been shown that DTs improved the prediction of response to neoadjuvant therapy in breast cancer. $^{7}$ The main advantage of DTs is their interpretability. Moreover, their extracted conditional rules can be used for developing expert systems.

The aim of this study was to develop and compare the performance of DT models for prediction of short-term mortality risk in patients who undergo on-pump CABG surgery with or without concomitant valve replacement surgery. We also compared their results with EuroSCORE, ${ }^{4}$ which is the most practical existing method in cardiac surgery risk assessment. 


$$
\begin{aligned}
& \text { Abbreviations and Acronyms } \\
& \text { ANN }=\text { artificial neural networks } \\
& \text { AUC }=\text { area under the curve } \\
& \text { CABG }=\text { coronary artery bypass grafting } \\
& \text { DT }=\text { decision tree } \\
& \text { EECDT }=\text { entropy error crisp decision tree } \\
& \text { EEFDT }=\text { entropy error fuzzy decision tree } \\
& \text { ICU }=\text { intensive care unit } \\
& \text { LR }=\text { logistic regression } \\
& \text { MAE }=\text { mean absolute error } \\
& \text { ROC }=\text { receiver operating characteristic }
\end{aligned}
$$

\section{PATIENTS AND METHODS}

The protocol of this study was approved by the local ethical committee of the medical faculty at Tehran University of Medical Sciences. The need for informed consent was waived.

\section{Patient Population and Data Collection}

The dataset includes the information for 1068 consecutive patients who were referred for CABG surgery at Rajaie Cardiovascular Medical and Research Center between September 2005 and April 2006. The study was mainly designed to develop a regression model to predict mortality and assess the quality of medical care among different surgeons and institutions. ${ }^{8}$ Patients with incomplete information on any predictive variable or outcome were excluded from this study. Postoperative variables were excluded from the multivariate analysis because new models can predict the preoperative mortality risk.

\section{Outcome}

The primary outcome was 30 -day postoperative mortality. This included all patients who died either at the hospital or within 30 days of the operation date. Follow-up took place at an outpatient clinic or by telephone interview. Postoperative data were length of intensive care unit (ICU) stay and major complications after surgery including myocardial infarction, cardiac output state, prolonged ventilation, central nervous system complications, serious infections, and oliguria or anuria.

\section{Statistical Analysis}

Continuous variables are summarized by means \pm standard deviation and categorical variables are expressed as proportions (\%). Univariate analyses were performed by either $\chi^{2}$ or Student $t$ test where applicable. Model development and statistical analyses were performed using MATLAB software (V7.8, R2009A) and SPSS statistical software (version 15.0.0; SPSS Inc, Chicago, Ill).

\section{Decision Tree Model}

DT modeling is a popular reasoning method and has been successfully used in medical decision making. DTs consist of multilayer connected nodes and each branch of a DT from root node to a terminal node results in an individual conditional rule (Appendix E1). In this study, we developed and compared fuzzy ${ }^{9}$ and crisp DTs $s^{9}$ to predict early mortality risk in patients. Fuzzy decision tree model. The fuzzy DT model applies fuzzy reasoning methods ${ }^{10}$ to be able to solve real-world problems more precisely. Therefore, this kind of regression tree uses a fuzzy discriminator function ${ }^{9}$ and predicts the degree of membership of each object to the outcome classes.

Survivor and no survivor were 2 outcome classes in this study. We have implemented an entropy error fuzzy decision tree (EEFDT) that applies both entropy ${ }^{11}$ and error ${ }^{9}$ functions to predict the degree of membership of each patient to each outcome class. Based on the method of Olaru and colleagues, ${ }^{9}$ EEFDT implementation consisted of 3 steps: growing, pruning, and refitting steps.

In the growing step, a fuzzy discriminator function found the best fuzzy cut point for each continuous variable. The best predictive variable (among continuous and categorical variables) was selected at each node to reduce the entropy of the dataset at that node (the gain ratio of all continuous and categorical variables was calculated at that node and the variable with the highest gain ratio was selected). ${ }^{11}$ This method continued iteratively until termination criteria ${ }^{9}$ were met (a growing step was terminated when the cardinality of the local node reached $\leq 10$ or the entropy of the local node reached $\leq 0.1$ or selecting none of the potential variables resulted in a gain ratio $\geq 0.01$ ). In the pruning step, the irrelevant parts of the grown tree were deleted to increase its interpretability. ${ }^{9}$ The nodes were deleted one by one in bottom-up order and the mean absolute error (MAE) of new subtrees was calculated. ${ }^{9}$ The best pruned subtree was the smallest one whose MAE was equal to min(MAE) + standard error of MAE that was estimated on the pruning set. ${ }^{9}$ In the refitting step, the parameters of the terminal nodes were optimized once more to reduce the error of grown and pruned trees. ${ }^{9}$

Crisp decision tree model. In this study, we also developed an entropy error crisp decision tree (EECDT) to predict early mortality risk. The implementation of this DT was the same as EEFDT. However, EECDT used a crisp discriminator function ${ }^{9}$ to find the best cut point for continuous variables in the growing step.

Both DTs were implemented and tested with 3 mutually exclusive subsets. Therefore, the whole dataset was randomly partitioned into 3 subsets: growing, pruning, and testing sets. The sets were stratified, which means that the proportion of dead cases in each dataset was kept around $4 \%$ in all sets. The tree was developed with growing, pruned with pruning, refitted with both growing and pruning, and tested with the testing set.

Logistic regression risk model. This model was developed by a combination of the pruning and refitting sets (two-thirds of the dataset) and was tested by the testing set (one-third of the dataset). Based on the full model approach, all predictive variables were retained to develop the model regardless of their statistical significance. A multivariate logistic regression (LR) analysis was then performed to evaluate the independent role of each variable, using probability values of .05 as the threshold for entering variables. In this method, we did not omit the predictive variables that are not statistically significant in the univariate analysis because they could achieve significance when other factors are included in the model. ${ }^{12}$ Significant independent variables were entered into the final model and the weight of each variable was obtained from the logistic $\beta$ coefficient.

\section{Model Performance}

The estimates from such a single hold-out cross validation, in which the dataset is partitioned into just 2 mutually exclusive subsets, is somehow biased and depends on the division of the training and testing sets. To get an estimate with lower bias and with potentially better predictive power of our method, we conducted another experiment. We repeated this holdout cross validation 10 times and the performance of all models was estimated by averaging. Accuracy, AUC, sensitivity, and specificity of the model were reported to evaluate their performance. The method proposed by Vergara and colleagues ${ }^{13}$ was used to evaluate the statistical difference between the AUC of the models.

Moreover, EuroSCORE ${ }^{4}$ was estimated for patients in the testing set in each hold-out cross validation circle. Accuracy, AUC, sensitivity, and specificity of this model were also calculated by averaging the results.

\section{RESULTS}

\section{Patient Characteristics}

A total of 1068 adult patients underwent CABG surgery between September 2005 and April 2006 at our institution. 
TABLE 1. Patient characteristics

\begin{tabular}{|c|c|c|c|}
\hline Variables & All patients (mean \pm SD) or $(\%)$ & Survivors $($ mean \pm SD) or $(\%)$ & No survivors $($ mean \pm SD) or $(\%)$ \\
\hline Height, m & $1.66 \pm 0.09$ & $1.67 \pm 0.09$ & $1.62 \pm 0.09$ \\
\hline Weight, kg & $80.91 \pm 11.60$ & $74 \pm 11.55$ & $68 \pm 12.46$ \\
\hline Hemoglobin, g/dL & $14.57 \pm 1.41$ & $13.10 \pm 1.40$ & $12.40 \pm 1.53$ \\
\hline Creatinine, $\mathrm{mg} / \mathrm{dL}$ & $1.42 \pm 0.79$ & $1.10 \pm 0.78$ & $1.20 \pm 0.85$ \\
\hline \multicolumn{4}{|l|}{ Age } \\
\hline $45-60$ y & 58.1 & 58.7 & 38.9 \\
\hline $61-75$ y & 39.5 & 38.9 & 57.3 \\
\hline$>76 \mathrm{y}$ & 2.4 & 2.4 & 3.8 \\
\hline \multicolumn{4}{|l|}{ Sex } \\
\hline Male & 86 & 87.2 & 55.6 \\
\hline Female & 14 & 12.8 & 44.4 \\
\hline \multicolumn{4}{|l|}{ Left ventricular ejection fraction } \\
\hline$<25 \%$ & 3.2 & 3.1 & 2.8 \\
\hline $25-45 \%$ & 62.6 & 62.6 & 66.6 \\
\hline$>45 \%$ & 34.2 & 34.3 & 30.6 \\
\hline \multicolumn{4}{|l|}{ Educational level } \\
\hline Illiterate & 21.5 & 21.7 & 16.7 \\
\hline High school & 74.1 & 73.8 & 83.3 \\
\hline University & 4.4 & 4.5 & 0 \\
\hline Substance abuse & 39.8 & 41 & 8.3 \\
\hline Hypertension & 38.9 & 38.3 & 55.6 \\
\hline Diabetes & 27 & 26.3 & 44.4 \\
\hline Carotid disease & 1.2 & 1.1 & 2.8 \\
\hline Chronic renal failure & 1.6 & 1.6 & 0 \\
\hline Chronic obstructive pulmonary disease & 1.3 & 1.2 & 2.8 \\
\hline Previous vascular surgery & 0.1 & 0 & 2.8 \\
\hline Claudication & 0.9 & 0.1 & 0 \\
\hline Cerebrovascular accident & 0.9 & 0.1 & 0 \\
\hline Other organ disease & 1.1 & 1.1 & 0 \\
\hline Previous CABG & 1.1 & 1.1 & 5.6 \\
\hline $\begin{array}{l}\text { Percutaneous transluminal coronary } \\
\text { angioplasty on admission }\end{array}$ & 0.8 & 0.8 & 0 \\
\hline Recent myocardial infarction & 12.1 & 11.8 & 19.4 \\
\hline Unstable angina & 15.3 & 14.9 & 25 \\
\hline Family history of coronary artery disease & 0.1 & 0.1 & 0 \\
\hline Postinfarction septal rupture & 0.1 & 0 & 2.8 \\
\hline Active endocarditis & 0 & 0 & 0 \\
\hline \multicolumn{4}{|l|}{ Angiographic data } \\
\hline Left main & 11.8 & 11.4 & 22.2 \\
\hline Left anterior descending & 89.9 & 89.8 & 91.7 \\
\hline Left circumflex & 56.3 & 56 & 63.9 \\
\hline Right coronary artery & 74.3 & 74.3 & 72.2 \\
\hline Obtuse marginal & 37 & 37.5 & 22.2 \\
\hline Diagonal & 40.1 & 40.7 & 25 \\
\hline Critical situation & 0.9 & 0.7 & 5.6 \\
\hline Distal coronary anastomosis & 0.1 & 0.1 & 0 \\
\hline \multicolumn{4}{|l|}{ CABG } \\
\hline Alone & 92.2 & 93.3 & 72.2 \\
\hline Aortic surgery & 0.1 & 0.8 & 5.6 \\
\hline Mitral valve & 2.2 & 2.1 & 5.6 \\
\hline Replacement aortic valve & 1.3 & 1 & 8.3 \\
\hline Replacement tricuspid valve & 0 & 0 & 0 \\
\hline Replacement other & 1.8 & 1.4 & 11.1 \\
\hline
\end{tabular}


TABLE 1. Continued

\begin{tabular}{|c|c|c|c|}
\hline Variables & All patients $($ mean \pm SD) or $(\%)$ & Survivors $($ mean \pm SD) or $(\%)$ & No survivors $($ mean \pm SD) or $(\%)$ \\
\hline \multicolumn{4}{|l|}{ Indication of surgery } \\
\hline Emergency & 3 & 2.9 & 5.6 \\
\hline Urgent & 3.6 & 2.4 & 16.7 \\
\hline Elective & 93.4 & 91.7 & 77.7 \\
\hline Catheterization laboratory complication & 0.2 & 0.1 & 2.8 \\
\hline \multicolumn{4}{|l|}{ Intensive care unit stay } \\
\hline$<2 \mathrm{~d}$ & 57.1 & 56.9 & 69.2 \\
\hline $3-6 \mathrm{~d}$ & 40.7 & 41.2 & 7.7 \\
\hline$>7 \mathrm{~d}$ & 2.2 & 1.9 & 23.1 \\
\hline \multicolumn{4}{|l|}{ Major complications } \\
\hline Myocardial infarction & 0.6 & 0.3 & 8.3 \\
\hline Cardiac output state & 5.8 & 5.1 & 25 \\
\hline Prolonged ventilation & 2.8 & 1.9 & 25 \\
\hline Central nervous system & 1.1 & 0.7 & 11.1 \\
\hline Serious infections & 0.4 & 0.2 & 5.6 \\
\hline Oliguria/anuria & 1.2 & 0.5 & 16.7 \\
\hline
\end{tabular}

$S D$, Standard deviation; $C A B G$, coronary artery bypass grafting.

Data for 120 patients $(11.2 \%)$ were excluded because of missing values for any predictive variable. Therefore, data from 948 patients were recruited for statistical analysis and model development (815 male patients [86\%] and 133 female patients $[14 \%])$. The total mortality rate was $3.8 \%$ (36 patients), which was significantly higher $(P<.001)$ in women $(12 \%)$ than men $(2.5 \%)$. Moreover, patients were categorized into 3 age groups (45-60 years, $61-75$ years, $\geq 76$ years); only 22 patients $(2.3 \%)$ were older than 76 years. Table 1 shows the categorical and continuous characteristics of the patients.

Patients' postoperative ICU stay was divided into 3 groups: less than 2 days, 3 to 6 days, and more than 7 days. ICU stay less than 2 days was not statistically different between survivor and no survivor groups, ICU stay for 3 to 6 days was statistically higher in survivors $(P<.001)$ and ICU stay more than 7 days was statistically higher in no survivors $(P<.001)$. Furthermore, the frequency of all postoperative complications was higher among no survivors than survivors $(P<.001)$ except oliguria or anuria, which were not statistically different $(P=.46)$ between the 2 groups.

\section{Fuzzy Decision Tree Model}

For developing DTs, the dataset was randomly partitioned into 3 mutually independent subsets. The model was grown with $350(37 \%)$, pruned with $300(31.6 \%)$, refitted with $650(68.6 \%)$, and tested with $298(31.4 \%)$ patients' data.

The best final EEFDT model selected 19 predictive variables and resulted in a tree with 39 nodes and 20 conditional rules. This model resulted in an AUC of $0.90 \pm 0.008$ and sensitivity and specificity of $75 \%$ and $98.6 \%$, respectively. The best final EECDT model selected 15 predictive variables and resulted in a tree with 35 nodes and 18 conditional rules. This model resulted in an AUC of $0.86 \pm 0.008$ and sensitivity and specificity of $58.3 \%$ and $97.4 \%$, respectively. Table 2 presents the corresponding detailed results of the final models. Figure $1, A$ and $B$, shows the final EEFDT and EECDT, respectively.

In each run of cross validation, 650 training data and 298 testing data were randomly selected so that the proportion of dead cases in each dataset was kept around 4\%. The average AUC, sensitivity, and specificity on the test set for EEFDT were $0.91 \pm 0.01,0.74 \pm 0.01$, and $0.98 \pm$ 0.016 , respectively, and for EECDT were $0.85 \pm 0.02$, $0.60 \pm 0.034$, and $0.98 \pm 0.01$, respectively.

\section{Logistic Regression Model}

A multivariate LR model was developed using 650 patients' data $(68.6 \%)$ and tested using 298 patients' data $(31.4 \%)$. Forty-four categorical and continuous predictive variables were imported into the model. The final regression model resulted in delineation of the optimal set of variables shown in Table 3 (constant $=0.289$ ); its overall performance is summarized in Table 2. The AUC was 0.78 (standard error $=0.008$ ) and with a probability threshold of .66 , the sensitivity and specificity were $25 \%$ and $99.7 \%$, respectively.

EuroSCORE ${ }^{4}$ is a well-known risk assessment model with its basic assumption based on LR. It showed an AUC

TABLE 2. The performance of the entropy error fuzzy decision tree (EEFDT), entropy error crisp decision tree (EECDT), logistic regression (LR) model, and EuroSCORE

\begin{tabular}{lccccc}
\hline & AUC & Accuracy & $\begin{array}{c}\text { Cut } \\
\text { point }\end{array}$ & $\begin{array}{c}\text { Sensitivity } \\
(\%)\end{array}$ & $\begin{array}{c}\text { Specificity } \\
(\%)\end{array}$ \\
\hline EEFDT & $0.90 \pm 0.008$ & 0.98 & 0.3 & 75 & 98.6 \\
EECDT & $0.86 \pm 0.008$ & 0.95 & 0.3 & 58.3 & 97.4 \\
LR & $0.78 \pm 0.008$ & 0.97 & 0.65 & 25 & 99 \\
EuroSCORE & $0.77 \pm 0.003$ & 0.96 & 0.11 & 3 & 100 \\
\hline
\end{tabular}

AUC, Area under the curve. 


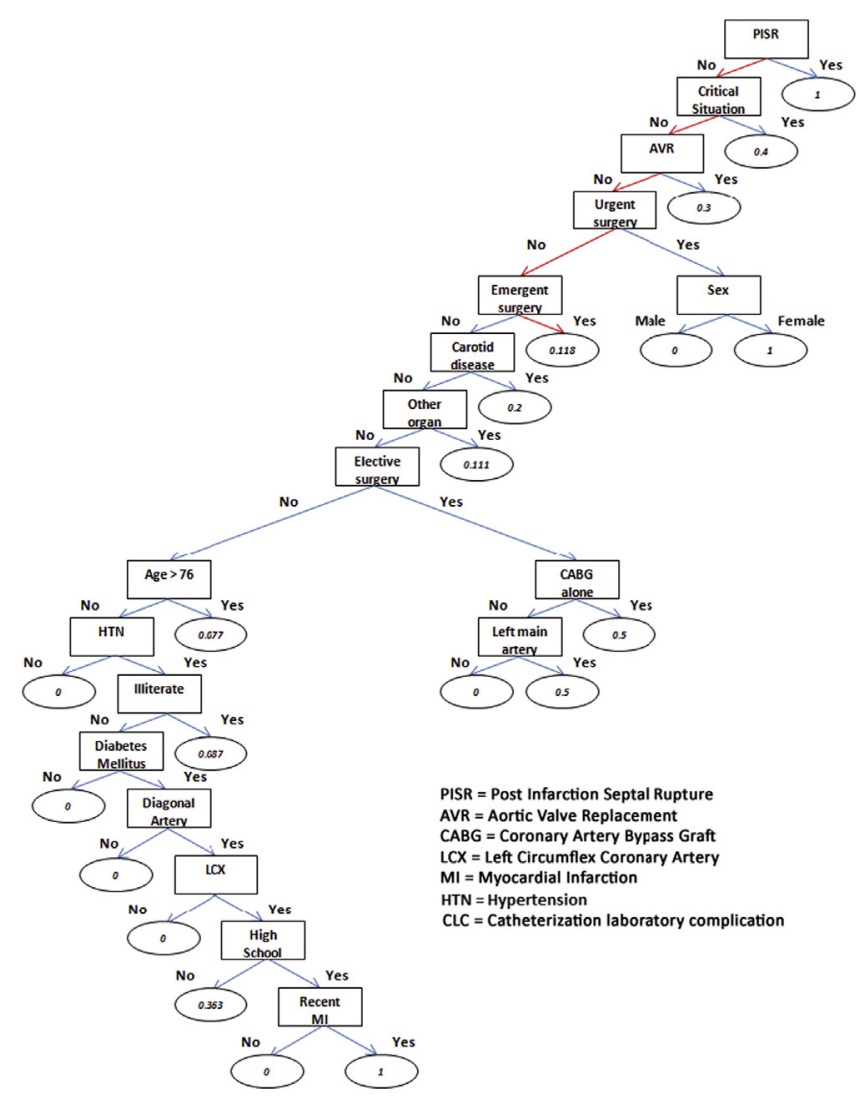

A

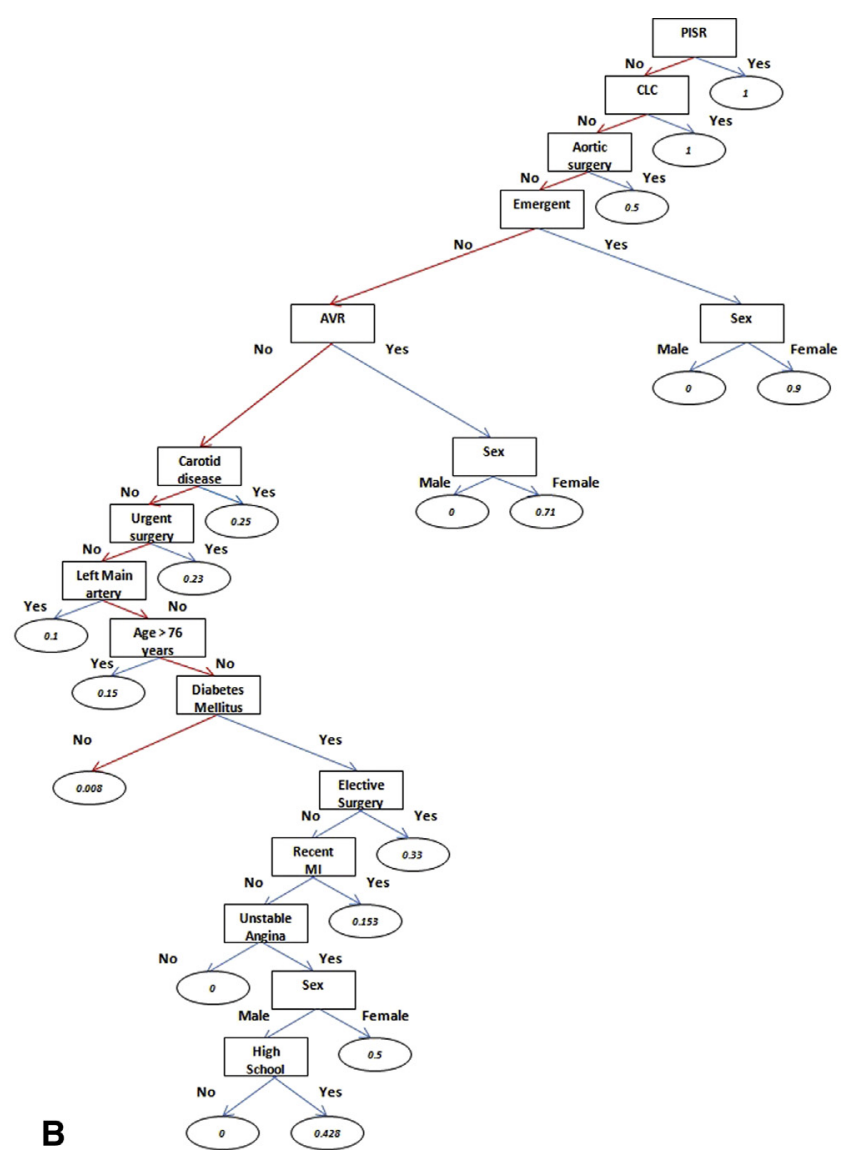

FIGURE 1. A, The final entropy error fuzzy decision tree; B, the final entropy error crisp decision tree.

of $0.77 \pm 0.003$ and with a probability threshold of .11 , the sensitivity and specificity were $3 \%$ and $100 \%$, respectively.

The average AUC, sensitivity, and specificity on the test sets (10 times cross validation) for the LR model were $0.80 \pm 0.03,0.26 \pm 0.02$, and $0.98 \pm 0.026$, respectively and for EuroSCORE were $0.78 \pm 0.02,0.04 \pm 0.01$, and $0.99 \pm 0.01$, respectively.

\section{DTs Versus Logistic Regression Risk Model}

Selected predictive variables in each model are shown in Table 3. Current practice in cardiac surgery is to conclude that a model discriminates well if the AUC is greater than 0.7. ${ }^{12}$ Therefore, all DTs, LR models, and EuroSCORE are acceptable in practice. Using Vergara and colleagues ${ }^{13}$ method, the AUC for EEFDT and EECDT were statistically higher than for the LR model and EuroSCORE $(P<.05)$ but were not statistically different $(P=.066$ and $P=.46$, respectively). Figure 2 shows the ROC curves of the final models.

\section{DISCUSSION}

In this study, we have used EEFDT and EECDT to predict the short-term mortality risk in CABG surgery. The results show that the performance of both models is superior compared with LR analysis and EuroSCORE. Moreover, the low sensitivity and high specificity of all methods can be related to class mismatch ( $4 \%$ mortality), which means that such models are less reliable for detecting patients with a high mortality risk. Both EEFDT and EECDT have significantly higher sensitivity than EuroSCORE and the LR model. Moreover, the sensitivity and specificity of EEFDT was higher than EECDT. Therefore, EEFDT is better than the other methods for detecting patients with a high mortality risk.

To our knowledge, our study is one of the few to report the application of DTs to predict the operative mortality of cardiac surgery. However, previous similar studies did not describe the final structure of the trees developed ${ }^{14}$ and the focus of other studies was on predicting the mortality rate of patients with both heart failure and acute myocardial infarction or patients who underwent a percutaneous coronary intervention. ${ }^{15,16}$

Extensive research has been done to develop applicable mortality risk models in cardiac surgery. ${ }^{3,4,17}$ But it is still difficult to predict risk for individual patients. ${ }^{18} \mathrm{LR}$ analysis is typically used to select the final set of risk factors; the Bayesian method ${ }^{17}$ and artificial neural networks $(\mathrm{ANN})^{19}$ have been used less commonly. Several studies 
TABLE 3. Selected predictive variables in the entropy error fuzzy decision tree (EEFDT), entropy error crisp decision tree (EECDT), and the logistic regression (LR) model with $\beta$ coefficient, odds ratio, standard error, and $P$ values for selected variables in the LR model

\begin{tabular}{|c|c|c|c|c|c|c|c|}
\hline & EEFDT & EECDT & LR & Coefficient & Odds ratio & Standard error & $P$ value \\
\hline \multicolumn{8}{|l|}{ Age } \\
\hline $45-60$ y & & & レ & -0.216 & 0.81 & 0.064 & .001 \\
\hline $61-75$ y & & & レ & -0.202 & 0.81 & 0.065 & .002 \\
\hline$\geq 76 \mathrm{y}$ & $レ$ & レ & 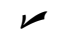 & -0.243 & 0.78 & 0.084 & .004 \\
\hline Carotid disease & レ & レ & レ & 0.181 & 1.2 & 0.90 & .046 \\
\hline Previous vascular surgery & & & レ & 0.858 & 2.36 & 0.172 & $<.001$ \\
\hline PISR & レ & レ & レ & 0.693 & 1.2 & 0.197 & .001 \\
\hline Aortic surgery & & $レ$ & & - & - & - & - \\
\hline \multicolumn{8}{|l|}{ Angiography cut-off } \\
\hline LAD & レ & $\nu$ & $\boldsymbol{V}$ & 0.074 & 1.1 & 0.035 & .033 \\
\hline Left main & レ & & & - & - & - & - \\
\hline Diagonal & レ & & & - & - & - & - \\
\hline LCX & & & & - & - & - & - \\
\hline \multicolumn{8}{|l|}{ CABG } \\
\hline Alone & レ & & & - & - & - & - \\
\hline$+\mathrm{AVR}$ & $\nu$ & 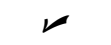 & $\boldsymbol{\nu}$ & 0.543 & 1.72 & 0.105 & $<.001$ \\
\hline CLC & & $\nu$ & 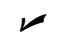 & 0.496 & 1.64 & 0.132 & $<.001$ \\
\hline Critical situation & $\nu$ & & & - & - & - & - \\
\hline \multicolumn{8}{|l|}{ Condition } \\
\hline Elective & $\nu$ & 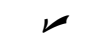 & & - & - & - & - \\
\hline Urgent & $\nu$ & $\nu$ & $\boldsymbol{\nu}$ & 0.196 & 1.2 & 0.082 & .017 \\
\hline Emergency & $\nu$ & $\nu$ & & - & - & - & - \\
\hline Recent MI & レ & 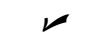 & & - & - & - & - \\
\hline Other organ disease & レ & & & - & - & - & - \\
\hline Unstable angina & & レ & & - & - & - & - \\
\hline Hypertension & レ & & & - & - & - & - \\
\hline Diabetes mellitus & レ & レ & & - & - & - & - \\
\hline \multicolumn{8}{|l|}{ Education } \\
\hline Illiterate & レ & & & - & - & - & - \\
\hline High school & $\nu$ & $\nu$ & & - & - & - & - \\
\hline Sex & $\nu$ & $\nu$ & & - & - & - & - \\
\hline
\end{tabular}

PISR, Postinfarction septal rupture; $L A D$, left anterior descending; $L C X$, left circumflex; $C A B G$, coronary artery bypass graft; $A V R$, aortic valve replacement; $C L C$, catheterization laboratory complication; $M I$, myocardial infarction.

evaluated the external validation of these risk models ${ }^{20}$ and it has been shown that the EuroSCORE model ${ }^{4}$ has the highest discriminatory power. ${ }^{19,20}$

EuroSCORE consists of 2 forms (additive and logistic), which estimate in-hospital mortality using 17 risk factors. ${ }^{4}$ Several studies have evaluated the validity of EuroSCORE in off-pump and on-pump CABG surgery. ${ }^{21,22}$ Although the performance of this model has been demonstrated to be acceptable under various conditions, its accuracy level rarely exceeded an AUC of $0.78 .^{5,23}$ Our study also evaluated the performance of EuroSCORE in an Iranian population at a referral tertiary center. The results show that this risk score has an AUC of 0.77, which was better than a previous study. ${ }^{24}$

In this study, we have also developed our own LR analysis to compare the results of DT methods with the best known statistical method in mortality risk prediction studies. It has been shown that the performance of DT models was superior to both the proposed LR model and
EuroSCORE. ${ }^{4}$ However, the proposed LR analysis has some advantages compared with previous ones. The first is the method of validation. We split our dataset and used a mutually exclusive test dataset for internal validation of our model. In other studies, the developers first reported the goodness of fit of their model on a developmental dataset, which is the weakest form of validation. ${ }^{12}$ The second advantage is the number of selected risk factors (10 variables), which was significantly lower in our LR method compared with EuroSCORE, which has 17 predictive variables.

LR models work based on a previous assumption of a linear relationship between risk factors. The results showed that the relationship among mortality risk predictive variables could be better identified by nonlinear assumptions. Therefore, applying more complex mathematical models with the capacity for nonlinear relationship could improve the accuracy of risk models. ${ }^{25}$ ANNs is one of such methods that has been used so far. ${ }^{19,26}$ The best proposed ANN 


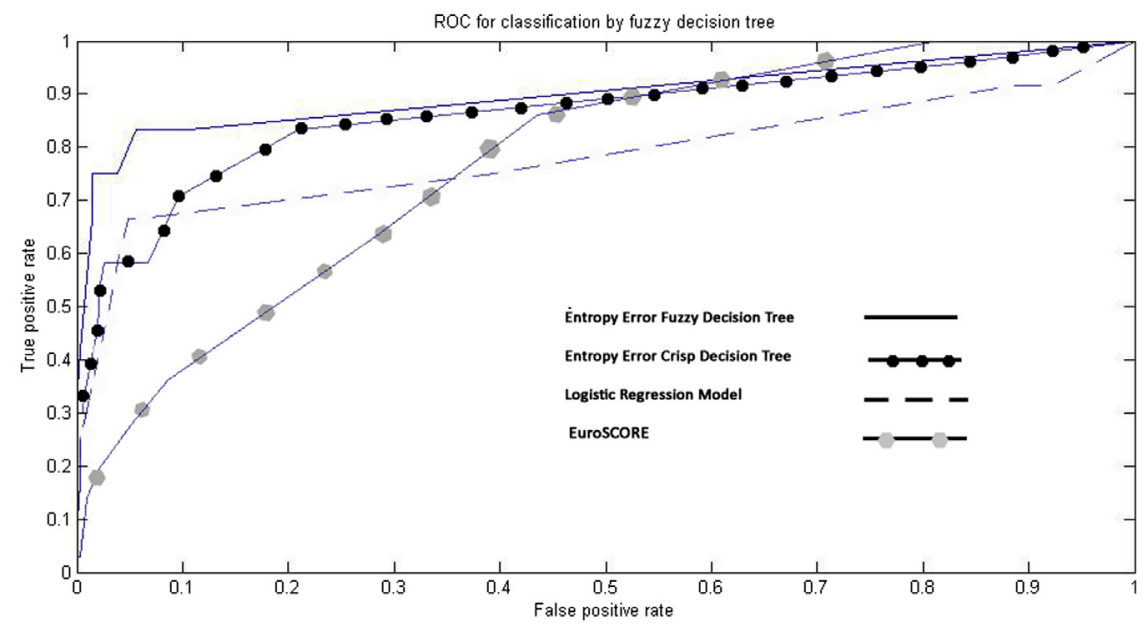

FIGURE 2. Receiver operating characteristic curves of the EEFDT, EECDT, LR model, and EuroSCORE.

selected 34 risk factors and resulted in an AUC of 0.81. ${ }^{19}$ Another study applied Bayes theorem. ${ }^{17}$ Despite their great accuracy, these models are not widely used because they are hard to design and require difficult calculations, often using dedicated software and computing knowledge that doctors do not welcome, besides being difficult to incorporate into clinical practice.

A DT is a kind of classifier that iteratively divides the dataset into subsets using 1 predictive variable at each node. The final DT has a hierarchical structure and easy to follow pathways (Figure 1) that are familiar to all clinicians. DTs have some advantages compared with other nonlinear methods. The first is their interpretability. Although they apply complex mathematical formula, they result in a limited number of conditional rules and a hierarchical algorithm that is clinically interpretable for patients and doctors. Moreover, the final DTs selected 15 and 19 predictive variables and their assessment is less time and resource consuming than the 34 variables in the ANN model.

\section{Limitations}

This study has some limitations. The first is the missing values. Although there are several statistical methods for substituting variables with missing values, we omitted them from our dataset and developed the models from patients' records with complete information on all risk factors and outcomes. It has been shown that the mortality rate for the data omitted was not statistically different to that in the applied dataset $(P=.47)$. Therefore, this may not have influenced the results.

The most stringent type of validation is external validation, which is superior to internal validation. ${ }^{12}$ In this study, we used a subset of patients' records from the same center to internally validate our models. Although Rajaie Cardiovascular Medical and Research Center is a referral institution for cardiac surgery, the performance of the model should be evaluated using a large, completely independent test dataset from various centers. ${ }^{27}$

Some unavoidable drawbacks exist that probably cause limitations in the discriminatory ability of all risk models. Some potential risk factors such as nutritional status or predilection to infections cannot be measured and entered into models. Furthermore, intraoperative and postoperative complications could influence the risk of mortality but cannot be considered preoperatively. ${ }^{28}$

In conclusion, our study shows that both EEFDT and EECDT are valuable new mathematical models in cardiac surgery risk assessment. Their performance and accuracy are superior compared with LR analysis and EuroSCORE. Based on the applicability and interpretability of the limited number of extracted conditional rules in DT models, they could be considered when counseling patients and comparing different surgeons and institutions. Further study is needed to assess the external validity of these proposed models.

\section{References}

1. Asimakopoulos G, Al-Ruzzeh S, Ambler G, Omar RZ, Punjabi P, Amrani M, et al. An evaluation of existing risk stratification models as a tool for comparison of surgical performances for coronary artery bypass grafting between institutions. Eur J Cardiothorac Surg. 2003;23:935-41; discussion 941-2.

2. Youn YN, Kwak YL, Yoo KJ. Can the EuroSCORE predict the early and mid-term mortality after off-pump coronary artery bypass grafting? Ann Thorac Surg. 2007;83:2111-7.

3. Shroyer AL, Coombs LP, Peterson ED, Eiken MC, DeLong ER, Chen A, et al The Society of Thoracic Surgeons: 30-day operative mortality and morbidity risk models. Ann Thorac Surg. 2003;75:1856-64; discussion 1864-5.

4. Nashef SA, Roques F, Michel P, Gauducheau E, Lemeshow S, Salamon R. European system for cardiac operative risk evaluation (EuroSCORE). Eur J Cardiothorac Surg. 1999;16:9-13.

5. D'Errigo P, Seccareccia F, Rosato S, Manno V, Badoni G, Fusco D, et al. Comparison between an empirically derived model and the EuroSCORE system in the evaluation of hospital performance: the example of the Italian CABG Outcome Project. Eur J Cardiothorac Surg. 2008;33:325-33.

6. Suner A, Celikoglu CC, Dicle O, Sokmen S. Sequential decision tree using the analytic hierarchy process for decision support in rectal cancer. Artif Intell Med. 2012;56:59-68. 
7. Takada M, Sugimoto M, Ohno S, Kuroi K, Sato N, Bando H, et al. Predictions of the pathological response to neoadjuvant chemotherapy in patients with primary breast cancer using a data mining technique. Breast Cancer Res Treat. 2012;134: 661-70.

8. Maleki M, Karbassi A, Noohi F, Omrani G. Risk factors and outcome in Iranian cardiac surgery: Iranian heart score. Am Heart Hosp J. 2007;5:223-7.

9. Olaru C, Wehenkel L. A complete fuzzy decision tree technique. Fuzzy Sets and Systems. 2003;138:221-54.

10. Zadeh LA. Fuzzy logic and approximate reasoning. Synthese. 1975;30:407-28.

11. Umanol M, Okamoto H, Hatono I, Tamura H, Kawachi F, Umedzu S, et al. Fuzzy decision trees by fuzzy ID3 algorithm and its application to diagnosis systems. In: Proceedings of the Third IEEE Conference on Fuzzy Systems. Vol. 3. 1994 IEEE World Congress on Computational Intelligence. IEEE; 1994:2113-8.

12. Omar RZ, Ambler G, Royston P, Eliahoo J, Taylor KM. Cardiac surgery risk modeling for mortality: a review of current practice and suggestions for improvement. Ann Thorac Surg. 2004;77:2232-7.

13. Vergara IA, Norambuena T, Ferrada E, Slater AW, Melo F. StAR: a simple tool for the statistical comparison of ROC curves. BMC Bioinformatics. 2008;9:265.

14. Rahman HAA, Bee Wah Y, Khairudin Z, Abdullah NN. Comparison of predictive models to predict survival of cardiac surgery patients. 2012 International Conference on Statistics in Science, Business, and Engineering (ICSSBE). IEEE; 2012:1-5.

15. Austin PC, Tu JV, Lee DS. Logistic regression had superior performance compared with regression trees for predicting in-hospital mortality in patients hospitalized with heart failure. J Clin Epidemiol. 2010;63:1145-55.

16. Negassa A, Monrad ES, Bang JY, Srinivas VS. Tree-structured risk stratification of in-hospital mortality after percutaneous coronary intervention for acute myocardial infarction: a report from the New York State percutaneous coronary intervention database. Am Heart J. 2007;154:322-9.

17. Marshall G, Shroyer AL, Grover FL, Hammermeister KE. Bayesian-logit model for risk assessment in coronary artery bypass grafting. Ann Thorac Surg. 1994; 57:1492-9; discussion 500 .
18. Pinna-Pintor P, Bobbio M, Colangelo S, Veglia F, Giammaria M, Çuni D, et al. Inaccuracy of four coronary surgery risk-adjusted models to predict mortality in individual patients. Eur J Cardiothorac Surg. 2002;21:199-204.

19. Nilsson J, Ohlsson M, Thulin L, Hoglund P, Nashef SA, Brandt J. Risk factor identification and mortality prediction in cardiac surgery using artificial neural networks. J Thorac Cardiovasc Surg. 2006;132:12-9.

20. Nilsson J, Algotsson L, Hoglund P, Luhrs C, Brandt J. Comparison of 19 preoperative risk stratification models in open-heart surgery. Eur Heart J. 2006; 27:867-74.

21. Nilsson J, Algotsson L, Hoglund P, Luhrs C, Brandt J. Early mortality in coronary bypass surgery: the EuroSCORE versus The Society of Thoracic Surgeons risk algorithm. Ann Thorac Surg. 2004;77:1235-9; discussion 1239-40.

22. Al-Ruzzeh S, Asimakopoulos G, Ambler G, Omar R, Hasan R, Fabri B, et al. Validation of four different risk stratification systems in patients undergoing off-pump coronary artery bypass surgery: a UK multicentre analysis of 2223 patients. Heart. 2003;89:432-5.

23. Zingone B, Pappalardo A, Dreas L. Logistic versus additive EuroSCORE. A comparative assessment of the two models in an independent population sample. Eur J Cardiothorac Surg. 2004;26:1134-40.

24. Sadeghi MM, Arasteh M, Gharipour M, Nilfroush P, Shamsolketabi H, Etesampour A, et al. Evaluation of accuracy of Euroscore risk model in prediction of perioperative mortality after coronary bypass graft surgery in Isfahan. J Res Med Sci. 2011;16:787-92.

25. Lippmann RP, Shahian DM. Coronary artery bypass risk prediction using neural networks. Ann Thorac Surg. 1997;63:1635-43.

26. Ennett CM, Frize M. Weight-elimination neural networks applied to coronary surgery mortality prediction. IEEE Trans Inf Technol Biomed. 2003; 7:86-92.

27. Altman DG, Royston P. What do we mean by validating a prognostic model? Stat Med. 2000; 19:453-73.

28. Nissinen J, Biancari F, Wistbacka JO, Peltola T, Loponen P, Tarkiainen P, et al. Safe time limits of aortic cross-clamping and cardiopulmonary bypass in adult cardiac surgery. Perfusion. 2009;24:297-305. 


\section{APPENDIX E1}

Figures 1 and 2 showed the final DTs in this study. The first node at the top is called the root node and it has no incoming edge. All other nodes with at least 1 outgoing edge are called test nodes (squares); the nodes without any outgoing edge are called terminal nodes (ovals). In EEFDT, the label on the terminal nodes demonstrates the degree of membership of that terminal node to the death class. This label means probability of death in EECDT as well.

Multiple pathways come down from the root node. Each pathway from the root node to each terminal node results in an individual conditional rule. For instance, the red pathway in Figure 1, $A$, results in an individual rule in the EEFDT model. This rule means that if the patient does not have postinfarction septal rupture, and is not in a critical situation, and does not need aortic valve replacement, and the surgery is not urgent, and the surgery is not an emergency, and the patient does not have carotid disease, and has other organ disease, then the degree of membership to class death is 0.111 . Therefore, the degree of membership to class survival is 0.889 .

Likewise, the red pathway in Figure $1, B$, results in an individual rule in the EECDT model. This rule means that if the patient does not have postinfarction septal rupture, and does not have catheterization laboratory complication, and does not need aortic surgery, and the surgery is not an emergency, and the patient does not need aortic valve replacement, and does not have carotid disease, and the surgery is not urgent, and the patient's angiography does not show cut-off in the left main coronary artery, and the patient age is not more than 76 years of age, and the patient does not have diabetes mellitus, that patient's probability of death is .008 .

One of the best advantages of DTs is their simple interpretable conditional rules. Although the results of DTs came from complex mathematical assumptions, like ANNs, their hierarchical architecture and easy to follow pathway would help clinicians to understand where such results came from and to interpret them in a medical manner. 